\title{
Non-invasive methods applied to the case of Municipal Solid Waste landfills (MSW): analysis of long-term data
}

\author{
A. Scozzari \\ CNR Institute of Geoscience and Earth Resources, Via Moruzzi 1, 56124 Pisa, Italy \\ Received: 7 July 2008 - Revised: 22 August 2008 - Accepted: 19 September 2008 - Published: 14 November 2008
}

\begin{abstract}
This work presents and discusses a methodology for modeling the behavior of a landfill system in terms of biogas release to the atmosphere, relating this quantity to local meteorological parameters. One of the most important goals in the study of MSW sites lies in the optimization of biogas collection, thus minimizing its release to the atmosphere.
\end{abstract}

After an introductory part, that presents the context of noninvasive measurements for the assessment of biogas release, the concepts of survey mapping and automatic flux monitoring are introduced.

Objective of this work is to make use of time series coming from long-term flux monitoring campaigns in order to assess the trend of gas release from the MSW site. A key aspect in processing such data is the modeling of the effect of meteorological parameters over such measurements; this is accomplished by modeling the system behavior with a set of Input/Output data to characterize it without prior knowledge (system identification).

The system identification approach presented here is based on an adaptive simulation concept, where a set of Input/Output data help training a "black box" model, without necessarily a prior analytical knowledge. The adaptive concept is based on an Artificial Neural Network scheme, which is trained by real-world data coming from a long-term monitoring campaign; such data are also used to test the real forecasting capability of the model.

In this particular framework, the technique presented in this paper appears to be very attractive for the evaluation of biogas releases on a long term basis, by simulating the effects of meteorological parameters over the flux measurement, thus enhancing the extraction of the useful information in terms of a gas "flux" quantity.

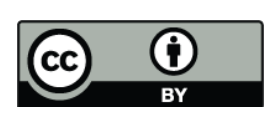

Correspondence to: A. Scozzari (a.scozzari@igg.cnr.it)

\section{Introduction}

Nowadays most of the human activities cause the production of industrial and municipal wastes. The landfilling has been up to now the most common technology for the management of such waste materials. One consequence of the landfill disposal is the production of biogas, due to the anaerobic degradation of organic matter by methanogenic bacteria. Biogas is a mixture of $\mathrm{CH}_{4}, \mathrm{CO}_{2}$, and other organic compounds that are present in traces, which often are toxic and malodorous. Besides, large part of the biogas generated by the landfills is responsible for the greenhouse effect.

Biogas leaks diffused by the soil/atmosphere interface are always present, even when a collection and combustion plant is active in the site. Since said leaks are generally a noticeable percentage of the total production of biogas, both energy recovery and environmental impact mitigation require the optimization of the biogas collection as a fundamental step to deal with.

With the aim of performing such optimization, there are various monitoring techniques available, such as:

- mapping the superficial distribution of biogas emissions

- thermal mapping of the landfill surface.

These mapping activities are necessary steps for planning any upgrade in the biogas collection and in the landfill coverage, as well as for checking the efficiency of both.

These tasks can be accomplished by the direct measurement of biogas fluxes coming from the surface with an accumulation chamber and the thermal mapping with a longwave infrared radiometer. Said activities are usually performed in the form of periodic field surveys.

A step ahead in these technologies can be represented by the usage of continuous monitoring systems, to collect long time series of flux and meteorological data at a certain location. Meteorological data are necessary in this context, due

Published by Copernicus Publications on behalf of the European Geosciences Union. 
to the high influence over the flux measurement. In fact, this work shows how, according to a system identification technique based on an Artificial Neural Network, the flux signal may be partially reconstructed by using weather information only. The model (predictor), adapted to a one-year long data series (training set), when applied to different periods, shows if the system is overperforming or underperforming with respect to the model, i.e. it shows how the overall efficiency of the system has changed with respect to the training set used to build up the predictor.

\section{Direct measurements of biogas releases at the inter- face with the atmosphere}

One of the main issues in the correct management of a landfill is the optimization of the biogas collection; this can be done via an adequate location of biogas wells, an efficient design of the extraction network and an appropriate usage of covering materials, all factors that contribute to the correct depressurization of the landfill body. The knowledge of the superficial distribution of biogas emission is the basis of energy recovery and environmental impact mitigation; this task is accomplished by using a device based on the accumulation chamber principle, which is preferred to the classical methods of soil gas survey, for a series of reasons whose explanation goes beyond the scope of this manuscript, and can be found in Raco et al. (2005).

In the proposed approach, direct measurements of biogas fluxes coming on the landfill surface are performed with an accumulation chamber instrumented with sensors selective towards carbon dioxide and/or methane, which are the two most abundant species rather easy to be measured with mature, portable devices.

In addition to that, known volatile toxic substances that are generally present in the biogas are carried by said carbon dioxide and methane.

The main purposes of these measurements are:

- to make an estimation of the total biogas output from the landfill soil,

- to compare the results of the direct measurements with the numerical models,

- to identify the areas having anomalous releases of biogas towards the atmosphere.

Such measurements can be performed in the following forms:

- periodic field surveys having an high spatial resolution,

- automatic continuous monitoring at a fixed location

\subsection{Survey mapping}

As stated above, the direct flux measurement has been chosen to obtain maps of biogas emissions in this context, and is usually employed to make periodic field surveys.

Due to the fact that the flux is measured in a series of punctual sampling points, most of the effort in processing the collected data lies in the management of a discrete dataset based on an irregular sampling grid to generate the following products:

- a superficial flux map (iso-flux map), defined on a continuous domain,

- the calculation of the total amount of biogas released to the atmosphere.

Without going further into geostatistical aspects, which are just given as a background information, what it is important to stress here is the importance of the processing of measured data to give overall estimations of biogas release and of the collection efficiency, that are essential parameters to assess the management of the waste disposal site and of its plants.

In particular, the calculation of the total biogas released to the atmosphere enables the validation of the collected gas measurement and the overall gas production model. Further details about the geostatistical techniques employed can be found in Krige (1951), Chauvet and Galli (1982).

In the application framework proposed in this work, data coming from the automatic monitoring activity are used for a better understanding and long-term assessment of the plant efficiency. The validation of this technique goes through the usage of survey mapping data according to the following main uses:

- to determine anomalous areas not related with the selected installation point of the automatic device

- to confirm the biogas production trend by calculating the total release.

In addition, the automatic continuous monitoring device presented in this work is based on the same working principle (accumulation chamber), that characterizes portable devices for field surveys.

\subsection{Automatic monitoring}

In addition to the measurement surveys based on multiple localized measurements, there's the possibility to install a steady automatic measurement station; the availability of data at a very high temporal resolution, at a given location, is complementary to the high spatial resolution, but very poor temporal one, obtained by periodic field surveys, such the ones described in the previous paragraph.

The automatic monitoring device is again based on the accumulation chamber principle and operates periodic flux measurements at the installation site. 


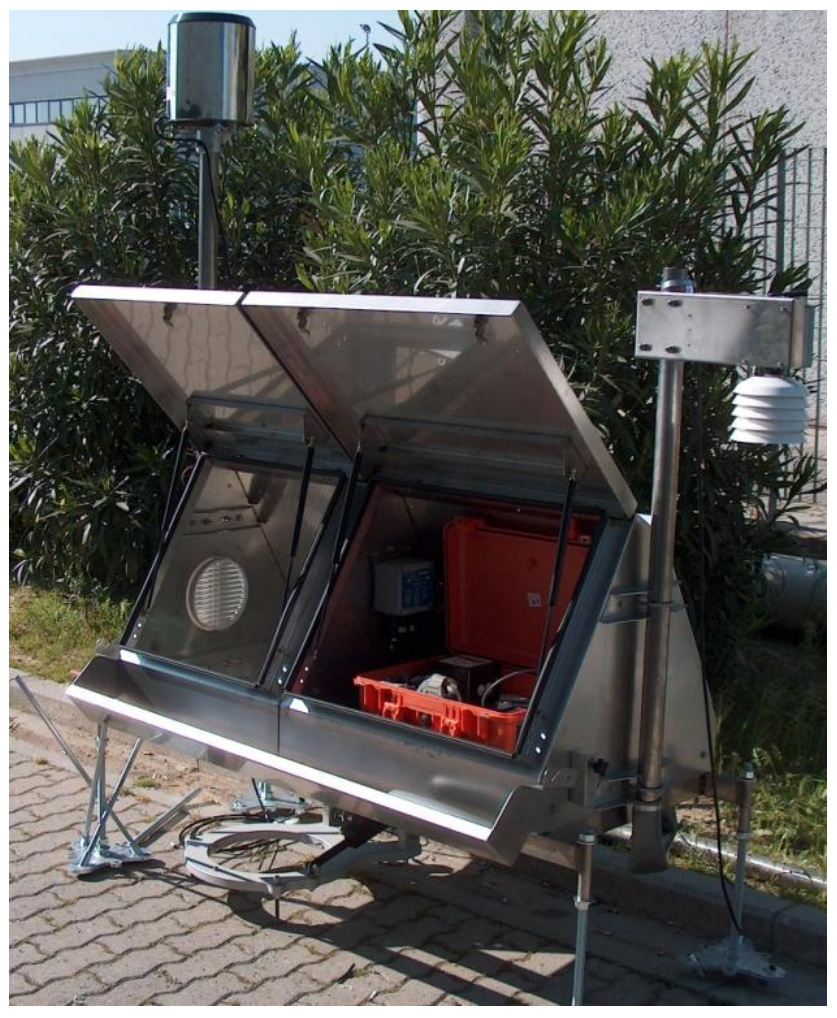

Fig. 1. The automatic measurement equipment as installed at the MSW site.

The approach based on this kind of instrument permits the following activities:

- to enhance the knowledge about how the external parameters (i.e. meteorological factors) modify the flux measurements,

- to assess the behavior of the landfill and its plants on a long term basis, providing monitoring information about how the waste disposal site develops to the management staff.

Figure 2 shows an overall view of the automatic measurement system before being installed, complete with the meteorological sensors.

\section{Long-term measurements: the role of a forecasting algorithm}

When dealing with long data series coming from a flux measurement station for long-term monitoring, particular attention must be given to the data processing aspects, especially in the evaluation of the influence of meteorological factors on such long-term measurements.

In fact, a complete meteorological station is associated to the automatic interfacial flux metering system. The combination of such data, as it often happens when dealing with

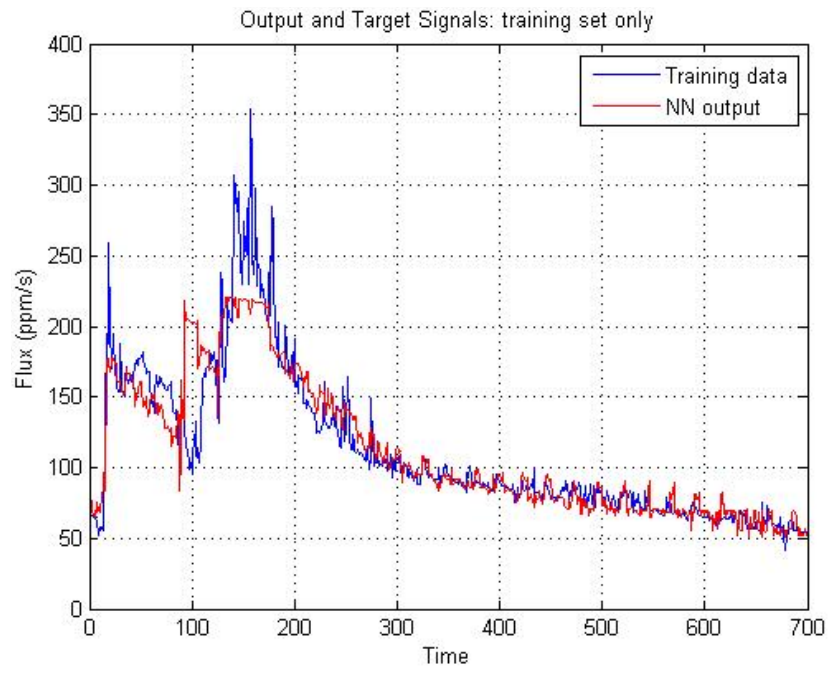

Fig. 2. Simulated and Output signals: focus on the transient due to the effect of rain.

multisensor techniques, offers the possibility to identify the system response to the various parameters involved in the observed process, in order to enhance the extraction of the desired information.

Under this respect, this work proposes a system identification methodology and an original application to the context of flux measurement data series.

\subsection{The dataset used}

Even if the approach described in this work may be considered as general, the availability of long time series coming from an experimental long-term monitoring, offers the possibility to present results in the form of a case study. In addition to that, the experience described here may represent an effective benchmark to assess the efficiency of adaptive techniques for the identification of the whole system.

More specifically, data have been taken from a longterm monitoring experiment concerning the MSW landfill of Legoli, which is located in Tuscany (Central Italy), about $30 \mathrm{Km}$ SE from the town of Pisa.

A data series that is approximately two years long (from July 2002 to July 2004), corresponding to a period of full activity of the waste disposal site, has been used. In fact, this MSW has been exploiting the collected biogas to produce electric energy since 1998, and has been substantially filled up during 2007.

An automatic monitoring station, installed at a fixed location in the MSW area, collects measurements of the biogas flux together with data about the state of the air and soil, completed by a set of standard meteorological parameters. The measured parameters are: $\mathrm{CO}_{2}$ flux from the soil, $\mathrm{CO}_{2}$ content in the air, air relative humidity, air temperature, 
atmospheric pressure, cumulated rain, wind speed and direction, soil temperature, soil moisture.

Data acquisition rate is one sample per hour, considered to be very adequate with respect to the natural speed of the observed phenomena; cumulated rain is calculated for each hour and added to the total; all the other parameters are averaged over the hour they refer to.

Part of the said parameters have a direct influence over the flux measurement, and the modelization of such effects is one essential aim of this work. In fact, the study of methods to de-trend flux data from meteorological artifacts or to predict the behavior of a system under some Input conditions, is a very useful step in building up techniques for the long term assessment of a process.

The dataset discussed here has been used to generate a model based on the measured data (system identification). The absence of a prior deterministic model for the process which is observed, requires a kind of black-box approach, where an Artificial Neural Network is trained to mimic the behavior of the observed system.

\subsection{The system identification approach}

Adaptive algorithms based on Artificial Neural Networks (ANN) are known in the literature for their capabilities in system identification (see Hu and Hwang, 2001, as a good example). Neural networks can be defined as asynchronous parallel processing systems, composed of a large number of simple interconnected elements, called neurons. Generally speaking, each neuron implements basic functions such as input weighting, signal summing and implementation of specific transfer functions (activation functions).

Different interconnection schemes and learning algorithms are known in the literature. When the architecture of a network has been defined, its internal coefficients (such as the interconnection weights) are determined in a learning phase, based on a specific dataset (training set), which is often carried out on a selected subset of the available data.

The Multi-Layer Perceptron network model used in this work, is a class of feed-forward networks with one ore more layers of neurons, called "hidden layers", between the Input and the Output layers. Internal connections between such layers are unidirectional from the Input to the Output of the network. This class of ANNs have proved to be very effective in the implementation of nonlinear Input/Output mapping functions.

A mathematical simulation of the Input/Output behavior of a system enables the forecasting of its Output in any possible Input condition to be simulated. This work shows the adaptive extraction of a model, which relates the biogas flux to meteorological parameters, applied to a long data series (about two years long) acquired at the Legoli MSW landfill as described in Sect. 3.1.
The first year of data has been used to identify the system (training set), and the obtained coefficients have been applied to the test sets as described later.

For the case example shown in this manuscript, system identification has been based on the following parameters:

- air temperature,

- atmospheric pressure,

- cumulated rain.

Even if these three parameters have not been selected according to some rigorous metric, to assess their degree of influence over the state of the system, these are reasonably the three most relevant parameters that influence said system behavior. Also, it must be recalled here that the underlying deterministic model of this process has little chance to be fully determined; this lack of knowledge suggests to resort to a system identification scheme where an ANN approximates the system by training it with a known Input/Output dataset.

The design of the network has been done under the following hypothesis:

- the system has memory, in the sense that its output is expected to be related to its past inputs and internal states,

- cumulated rain data are converted into a continuous signal according to a model that takes into account a function which approximates the memory effect related to the rain.

The second hypothesis leads to a blind deterministic identification approach (a good description can be found in Luo and Hunbehauen, 1997), where the input of the system due to the effect of rain is treated as an arbitrary deterministic signal, where a particular wave shape, thought to be reasonably close to the real system behavior, is chosen.

Cumulated rain is converted into instantaneous discrete values by differentiation. The effect of rain over the flux measurement is modeled as a first-order exponential decay, taken as a pulse-response of the whole system to the rain.

Thus, the effect of the rain is simulated by the convolution of said discrete values (taken as a sequence of Kronecker deltas modulated by the hourly amount of rain) with said pulse response, and fed to the ANN.

A Multi-Layer Perceptron Neural Network (MLP-NN) with 12 inputs has been experimented (Hu and Hwang, 2001). In fact, signals fed to the ANN are the present plus the last 3 values for each of the 3 parameters. The output is the flux value at the instant corresponding to the present values fed to the ANN. Acquisition rate is kept at 1 sample per hour as stated before.

The essential design features of the feed-forward MLP network are summarized as follows: 


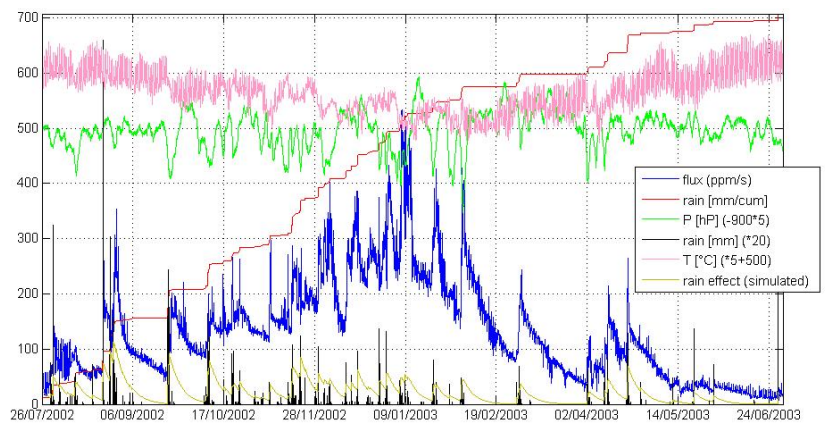

Fig. 3. Behavior of the flux and meteorological parameters during the period taken as training set of the network.

- one input layer with 12 inputs,

- one hidden layer with 29 neurons,

- hyperbolic tangent sigmoid transfer function for the hidden layer neurons,

- linear transfer function for the output neuron.

One example of the obtained result, shown for the time interval used to determine the time constant of the exponential decay model, is presented in Fig. 2. The blue line in the graph represents the system Output (flux), while the red line shows the value predicted by the ANN. The covered period is about 4 weeks long.

\section{Results}

The behavior of the flux and relevant meteorological data during the period taken as a training set of the ANN is shown in Fig. 3. This dataset, which regards the period between 26 July 2002 and 1 July 2003, has been used to train the ANN according to the Levenberg-Marquardt backpropagation algorithm, taking the flux data as the Output target.

The result is a trained network that, taking as an Input the time series of air temperature, air pressure and cumulated rain (after converting), predicts the corresponding value of the flux as an Output.

The evolution of a system may be assessed by applying the calculated model to different intervals of time, in order to understand how different is the reaction of the system to its Inputs, with respect to the model, in the selected time intervals.

Figure 4 shows the prediction of the flux, according to the ANN model, by using as a test set the same dataset used to train the network. The error variance in such estimation, which about $36 \mathrm{ppm} / \mathrm{s}$, is reflected by the good accordance between the measured data and the model.

Figure 5 shows the usage of the same model, trained on the previously described training set, applied to the dataset

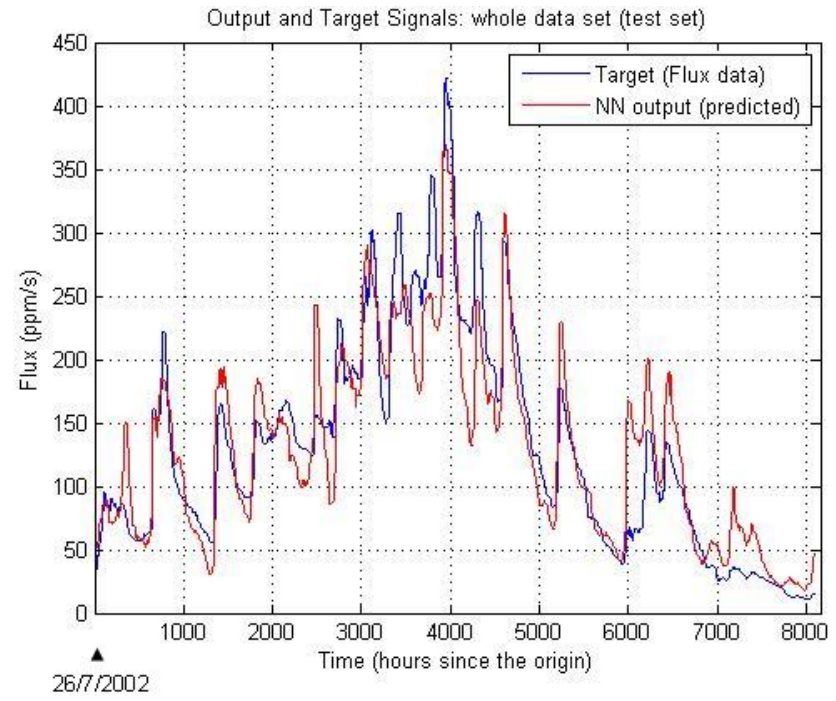

Fig. 4. Measured and predicted fluxes during the first year of the test set (26 July 2002 to 1 July 2003).

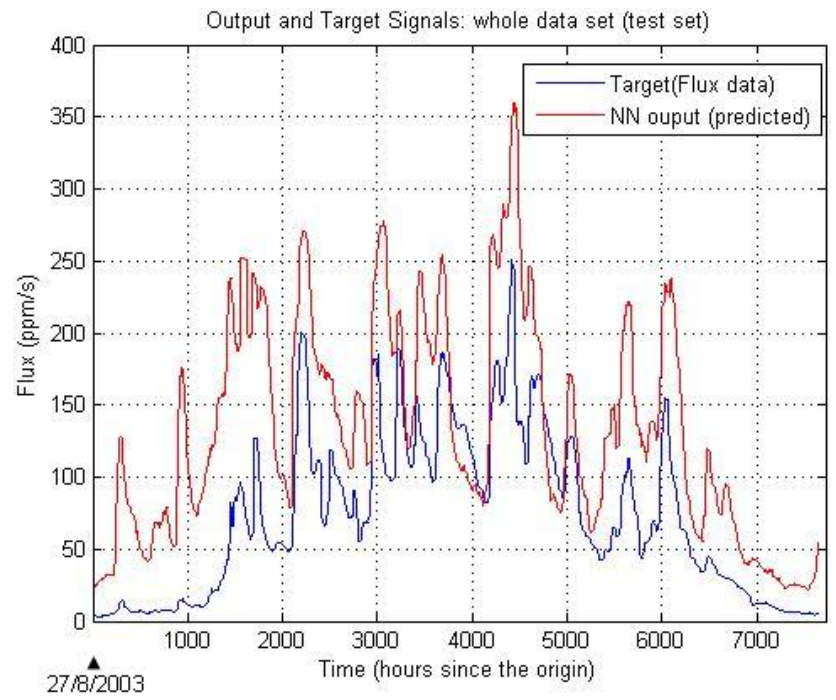

Fig. 5. Measured and predicted fluxes during the second year of the test set (27 August 2003 to 14 July 2004).

related to the second year of acquired data. The model Output shows an overestimation of predicted flux with respect to the acquired data.

It comes immediately clear that the measurement of the interfacial flux is very critical, since it is mostly explained by meteorological factors. This approach may also be used to partially de-trend the flux measurement from meteorological effects.

There is a seasonal underestimation of flux during winter, that appears when the test set corresponds to the training set (first year of data). The same adapted net applied to the second year dataset shows an overestimation all over the year. 
This result is qualitatively confirmed by landfill gas production data, that experienced an increment of collected biogas quantity by the plant during the second year of operation inside this dataset. This result may be an interesting overall indicator of the management quality, by comparing real emissions with forecasted ones.

A more quantitative approach in relating the prediction displacement with respect to measured emissions with biogas collection performance should be desirable, and gives room for future works.

\section{Conclusions}

The technique presented in this work appears to be very attractive for the evaluation of biogas releases on a long term basis, by simulating the effects of meteorological parameters as shown; in fact, after proving that meteorological parameters have a strong influence over the measured flux, so to be able to substantially reconstruct fluxes from meteorological parameters, a technique has been presented for the extraction of the useful information in terms of biogas flux according to the following scheme:

- evaluation of an adequate model with meteorological parameters at the Input and superficial gas flux at the Output,

- application of the model developed to different time intervals, to identify over/under estimations of flux with respect to measured data.

In this framework, overestimation means a lower release with respect to the model prediction, while underestimation implies an higher gas release with respect to the model.

Also, periodic seasonal effects are so evident that an efficient approach should be based on yearly data series, both for training (ANN model adaptation) and for testing (evaluation by applying the model).
In conclusion, an original application of Artificial Neural Networks to the context of soil flux measurements in landfill sites has been shown. The system identification capability of this kind of signal processing structures has been experimented, in order to make a model of the whole system reaction to a set of meteorological inputs. Results of this preliminary activity invite to a deeper study and application of this technique, aiming at a quantitative assessment of the biogas release related to the production state of the MSW site; under this point of view, the further development of this approach can provide an effective support to the analysis of long term data about emission monitoring, thus giving a better interpretation and usage of such data.

Acknowledgements. I first of all have to thank very much my collegue Brunella Raco for having the idea of this long-term monitoring and for providing the data discussed in this manuscript. Also, many thanks go to Belvedere SpA (the company which manages the Legoli landfill) for their constant availability and open-minded attitude.

Finally, the availability of these data is due to West Systems Srl, that is the manufacturer of the automatic monitoring device.

Edited by: F. Soldovieri

Reviewed by: two anonymous referees

\section{References}

Raco, B., Scozzari, A., Guidi, M., Lelli M., and Lippo, G.: Comparison of two non-invasive methodologies to monitor diffuse biogas emissions from MSW landfills soil: a case study, Sardinia Symposium 2005.

Krige, D. G.: A statistical approach to some basic mine valuation problems on the Witwatersrand, J. Chem. Metall. Min. Soc. S. Afr., 52, 119-139, 1951.

Chauvet, P. and Galli A.: Universal Kriging, Pubblication n. C - 96, Centre de Géostatistique, Ecole des Mines de Paris, Fontainebleau, 1982.

Luo, F. L. and Unbehauen, R.: Applied neural networks for signal processing, Cambridge Press, 1997.

Hu, Y. H. and Hwang, J. N.: Handbook of Neural Network Signal Processing, CRC Press, 2001. 